\title{
AGN feedback in the nucleus of M 51 (Corrigendum)
}

\author{
M. Querejeta ${ }^{1}$, E. Schinnerer ${ }^{1}$, S. García-Burillo ${ }^{2}$, F. Bigiel ${ }^{3}$, G. A. Blanc ${ }^{4,5,6}$, D. Colombo ${ }^{7}$, A. Hughes ${ }^{8,9}$, \\ K. Kreckel ${ }^{1}$, A. Leroy ${ }^{10}$, S. E. Meidt ${ }^{1}$, D. S. Meier ${ }^{11}$, J. Pety ${ }^{12,13}$, and K. Sliwa ${ }^{1}$ \\ 1 Max Planck Institute for Astronomy, Königstuhl, 17, 69117 Heidelberg, Germany \\ e-mail: querejeta@mpia-hd.mpg.de \\ 2 Observatorio Astronómico Nacional, Alfonso XII, 3, 28014 Madrid, Spain \\ 3 Institut für theoretische Astrophysik, Zentrum für Astronomie der Universität Heidelberg, Albert-Ueberle-Str. 2, \\ 69120 Heidelberg, Germany \\ ${ }^{4}$ Departamento de Astronomía, Universidad de Chile, 1515 Camino del Observatorio, Las Condes, Santiago, Chile \\ 5 Centro de Astrofísica y Tecnologías Afines (CATA), 1515 Camino del Observatorio, Las Condes, Santiago, Chile \\ 6 Visiting Astronomer, Observatories of the Carnegie Institution for Science, 813 Santa Barbara St, Pasadena, CA, 91101, USA \\ 7 Max Planck Institute for Radioastronomy, Auf dem Hügel 69, 53121 Bonn, Germany \\ 8 CNRS, IRAP, 9 Av. Colonel Roche, BP 44346, 31028 Toulouse, France \\ 9 Université de Toulouse, UPS-OMP, IRAP, 31028 Toulouse, France \\ 10 Department of Astronomy, The Ohio State University, 140 West 18th Avenue, Columbus, OH, 43210, USA \\ 11 Physics Department, New Mexico Institute of Mining and Technology, 801 Leroy Place, Socorro, NM, 87801, USA \\ 12 Institut de Radioastronomie Millimétrique, 300 rue de la Piscine, 38406 Saint Martin d'Hères, France \\ 13 Observatoire de Paris, 61 Avenue de l'Observatoire, 75014 Paris, France
}

A\&A 593, A118 (2016), DOI: 10.1051/0004-6361/201628674

Key words. galaxies: ISM - galaxies: active - galaxies: Seyfert - galaxies: structure - galaxies: jets - errata, addenda

The molecular gas outflow rates estimated in Sect. 3.3.1 of this paper contain a numerical error, and applying Eq. (1) to the reported measurements $\left(M_{\mathrm{H}_{2}}=4.1 \times 10^{6} M_{\odot}, M_{\text {dense }}=\right.$ $2.7 \times 10^{6} M_{\odot} ; R_{\text {out }} \sim 37 \mathrm{pc} ; V_{\text {out }} \sim 100 \mathrm{~km} \mathrm{~s}^{-1}$ ) leads to an estimate of $32 \tan (\alpha) M_{\odot} / \mathrm{yr}$ for the bulk molecular gas traced by $\mathrm{CO}(1-0)$, and $20 \tan (\alpha) M_{\odot} / \mathrm{yr}$ for the dense molecular gas traced by $\mathrm{HCN}(1-0)$. This is based on an idealised (multi)conical model, whose applicability to the outflow in M 51 was already questioned in the paper.

The final outflow rate estimates depend strongly on the angle $\alpha$, which is largely unconstrained. Under the assumption that the outflowing gas has the same inclination with respect to the disc as the one inferred for the radio plasma jet $\left(\alpha=70^{\circ}\right)$, this would result in a molecular outflow rate of $\sim 90 M_{\odot} / \mathrm{yr}$ $\left(\sim 55 M_{\odot} /\right.$ yr in the dense phase traced by the $\mathrm{HCN}(1-0)$ line); more moderate inclinations with respect to the line of sight lead to numerical values of a few times $10 M_{\odot} / \mathrm{yr}$. The originally quoted value of $\sim 1 M_{\odot} / \mathrm{yr}$ can only be recovered if the outflowing gas propagates very close to the line of sight.

Therefore, most probably, this instantaneous molecular outflow rate is larger than the secular inflow rate of $\sim 1 M_{\odot} / \mathrm{yr}$, whereas in the paper both rates were found to be roughly equal. We speculate that this is the result of the different timescales over which the inflow and outflow processes operate, suggesting that the molecular outflow is episodic, whereas the transport of gas from kpc-scales to the nucleus proceeds at a more steady rate.

The corrected outflow rate estimates also affect the kinetic luminosity and momentum flux calculated in Sect. 4.5, resulting in updated values of $L_{\text {kin }}=2.3 \times 10^{42} \mathrm{erg} / \mathrm{s}\left(1.5 \times 10^{42} \mathrm{erg} / \mathrm{s}\right.$ for the dense phase traced by $\mathrm{HCN})$, and $\mathrm{d} P / \mathrm{d} t=1.6 \times 10^{35} \mathrm{~g} \mathrm{~cm} \mathrm{~s}^{-2}$ $\left(1.0 \times 10^{35} \mathrm{~g} \mathrm{~cm} \mathrm{~s}^{-2}\right.$ for $\left.\mathrm{HCN}\right)$. The values of the kinetic energy $\left(E_{\text {kin }}\right)$ and momentum $(P)$ are not affected by the error in the calculation (but we emphasise that they rely on the assumption of $\alpha=70^{\circ}$, whereas the values quoted by Matsushita et al. (2007), are lower limits corresponding to an outflow expanding along the line of sight). These updated values do not change any of the qualitative conclusions in terms of energetics, except for the fact that M51 would no longer be a lower outlier in the outflow rates-bolometric luminosity compilation from García-Burillo et al. (2015).

We thank Stefano Carniani for pointing out this unfortunate mistake.

\section{References}

García-Burillo, S., Combes, F., Usero, A., et al. 2015, A\&A, 580, A35 Matsushita, S., Muller, S., \& Lim, J. 2007, A\&A, 468, L49 